\title{
Some properties of the Catalan-Qi function related to the Catalan numbers
}

Feng $\mathrm{Qi}^{1,2,4^{*}} \mathbb{C}$, Mansour Mahmoud ${ }^{3}$, Xiao-Ting Shi ${ }^{4}$ and Fang-Fang Liu ${ }^{4}$

*Correspondence: qifeng618@gmail.com ${ }^{1}$ Institute of Mathematics, Henan Polytechnic University, Jiaozuo City 454010, Henan Province, China Full list of author information is available at the end of the article

\begin{abstract}
In the paper, the authors find some properties of the Catalan numbers, the Catalan function, and the Catalan-Qi function which is a generalization of the Catalan numbers. Concretely speaking, the authors present a new expression, asymptotic expansions, integral representations, logarithmic convexity, complete monotonicity, minimality, logarithmically complete monotonicity, a generating function, and inequalities of the Catalan numbers, the Catalan function, and the Catalan-Qi function. As by-products, an exponential expansion and a double inequality for the ratio of two gamma functions are derived.
\end{abstract}

Keywords: Property, Catalan number, Catalan function, Catalan-Qi function, Asymptotic expansion, Integral representation, Logarithmic convexity, Complete monotonicity, Logarithmically complete monotonicity, Minimality, Inequality, Ratio of gamma functions

Mathematics Subject Classification: Primary 33B15; Secondary 05A10, 05A15, 05A16, 05A19, 05A20, 11B83, 11Y55, 11Y60, 26A48, 26A51, 26D15, 33C05, 44A20

\section{Background}

It is stated in Koshy (2009), Stanley and Weisstein (2015) that the Catalan numbers $C_{n}$ for $n \geq 0$ form a sequence of natural numbers that occur in tree enumeration problems such as "In how many ways can a regular $n$-gon be divided into $n-2$ triangles if different orientations are counted separately?" whose solution is the Catalan number $C_{n-2}$. The Catalan numbers $C_{n}$ can be generated by

$$
\begin{aligned}
\frac{2}{1+\sqrt{1-4 x}} & =\frac{1-\sqrt{1-4 x}}{2 x}=\sum_{n=0}^{\infty} C_{n} x^{n} \\
& =1+x+2 x^{2}+5 x^{3}+14 x^{4}+42 x^{5}+132 x^{6}+429 x^{7}+1430 x^{8}+\cdots .
\end{aligned}
$$

Two of explicit formulas of $C_{n}$ for $n \geq 0$ read that

$$
C_{n}=\frac{4^{n} \Gamma(n+1 / 2)}{\sqrt{\pi} \Gamma(n+2)}={ }_{2} F_{1}(1-n,-n ; 2 ; 1),
$$

where

$$
\Gamma(z)=\int_{0}^{\infty} t^{z-1} e^{-t} \mathrm{~d} t, \quad \mathfrak{R}(z)>0
$$

(c) 2016 The Author(s). This article is distributed under the terms of the Creative Commons Attribution 4.0 International License (http://creativecommons.org/licenses/by/4.0/), which permits unrestricted use, distribution, and reproduction in any medium, provided you give appropriate credit to the original author(s) and the source, provide a link to the Creative Commons license, and indicate if changes were made. 
is the classical Euler gamma function and

$$
{ }_{p} F_{q}\left(a_{1}, \ldots, a_{p} ; b_{1}, \ldots, b_{q} ; z\right)=\sum_{n=0}^{\infty} \frac{\left(a_{1}\right)_{n} \cdots\left(a_{p}\right)_{n}}{\left(b_{1}\right)_{n} \cdots\left(b_{q}\right)_{n}} \frac{z^{n}}{n !}
$$

is the generalized hypergeometric series defined for complex numbers $a_{i} \in \mathbb{C}$ and $b_{i} \in \mathbb{C} \backslash\{0,-1,-2, \ldots\}$, for positive integers $p, q \in \mathbb{N}$, and in terms of the rising factorials $(x)_{n}$ defined by

$$
(x)_{n}=\prod_{\ell=0}^{n-1}(x+\ell)= \begin{cases}x(x+1) \cdots(x+n-1), & n \geq 1 \\ 1, & n=0\end{cases}
$$

and

$$
(-x)_{n}=(-1)^{n}(x-n+1)_{n} .
$$

In Graham et al. (1994), Koshy (2009), Stanley and Weisstein (2015), Vardi (1991), it was mentioned that there exists an asymptotic expansion

$$
C_{x} \sim \frac{4^{x}}{\sqrt{\pi}}\left(\frac{1}{x^{3 / 2}}-\frac{9}{8} \frac{1}{x^{5 / 2}}+\frac{145}{128} \frac{1}{x^{7 / 2}}+\cdots\right)
$$

for the Catalan function $C_{x}$. What is the general expression for the asymptotic expansion (5)?

In Qi et al. (2015b, Remark 1) an analytical generalization of the Catalan numbers $C_{n}$ and the Catalan function $C_{x}$ was given by

$$
C(a, b ; z)=\frac{\Gamma(b)}{\Gamma(a)}\left(\frac{b}{a}\right)^{z} \frac{\Gamma(z+a)}{\Gamma(z+b)}, \quad \mathfrak{R}(a), \mathfrak{R}(b)>0, \quad \mathfrak{R}(z) \geq 0
$$

and the integral representation

$$
\begin{aligned}
C(a, b ; x)= & \frac{\Gamma(b)}{\Gamma(a)}\left(\frac{b}{a}\right)^{x} \frac{(x+a)^{x}}{(x+b)^{x+b-a}} \\
& \times \exp \left[b-a+\int_{0}^{\infty} \frac{1}{t}\left(\frac{1}{1-e^{-t}}-\frac{1}{t}-a\right)\left(e^{-a t}-e^{-b t}\right) e^{-x t} \mathrm{~d} t\right]
\end{aligned}
$$

for $a, b>0$ and $x \geq 0$ was derived. For uniqueness and convenience of referring to the quantity (6), we call $C(a, b ; x)$ the Catalan-Qi function. It is clear that

$$
C(a, b ; 0)=C(a, b ; 1)=1 \quad \text { and } \quad C(a, b ; x)=\frac{1}{C(b, a ; x)} .
$$

The integral representation (7) generalizes an integral representation for $C\left(\frac{1}{2}, 2 ; x\right)$ in Shi et al. (2015). Currently we do not know and understand the combinatorial interpretations of $C(a, b ; x)$ and its integral representation (7). Here we would not like to discuss the combinatorial interpretations of them. What we concern here is the asymptotic expansion similar to $(5)$ for $C(a, b ; x)$. 
In Koshy (2009) and from https://en.wikipedia.org/wiki/Catalan_number, the integral representation

$$
C_{n}=\frac{1}{2 \pi} \int_{0}^{4} \sqrt{\frac{4-x}{x}} x^{n} \mathrm{~d} x
$$

was listed. In Nkwanta and Tefera (2013, p. 10), there is an integral representation

$$
C_{n}=\frac{2^{2 n+5}}{\pi} \int_{0}^{1} \frac{x^{2}\left(1-x^{2}\right)^{2 n}}{\left(1+x^{2}\right)^{2 n+3}} \mathrm{~d} x
$$

In Qi et al. (2015c, Theorem 1.4), the integral representations

$$
C_{n}=\frac{1}{\pi} \int_{0}^{\infty} \frac{\sqrt{t}}{(t+1 / 4)^{n+2}} \mathrm{~d} t=\frac{2}{\pi} \int_{0}^{\infty} \frac{t^{2}}{\left(t^{2}+1 / 4\right)^{n+2}} \mathrm{~d} t, \quad n \geq 0
$$

was established. In Qi (2015a, Theorem 1.3), the equivalence relation between (8) and (9) was verified. What is the integral representation of the Catalan-Qi function $C(a, b ; x)$ similar to either (8) or (9)?

From the power series (1), we observe that the Catalan numbers $C_{n}$ is an increasing sequence in $n \geq 0$ with $C_{0}=C_{1}$. What about the monotonicity and convexity of the Catalan numbers $C_{n}$, the Catalan function $C_{x}$, and the Catalan-Qi function $C(a, b ; x)$ ? In Temme (1996, p. 67), it was listed that

$$
\frac{\Gamma(z+a)}{\Gamma(z+b)}=\frac{1}{\Gamma(b-a)} \int_{0}^{\infty}\left(1-e^{-u}\right)^{b-a-1} e^{-(z+a) u} \mathrm{~d} u, \quad b>a \geq 0 .
$$

Accordingly, we obtain an alternative integral representation

$$
C(a, b ; x)=\frac{1}{B(a, b-a)}\left(\frac{b}{a}\right)^{x} \int_{0}^{\infty}\left(1-e^{-u}\right)^{b-a-1} e^{-(x+a) u} \mathrm{~d} u
$$

for $b>a>0$ and $x \geq 0$, where $B(z, w)$ denotes the classical beta function which can be defined (Abramowitz and Stegun 1972, p. 258, 6.2.1 and 6.2.2) by

$$
B(z, w)=\int_{0}^{1} t^{z-1}(1-t)^{w-1} \mathrm{~d} t=\int_{0}^{\infty} \frac{t^{z-1}}{(1+t)^{z+w}} \mathrm{~d} t
$$

for $\mathfrak{R}(z)>0$ and $\mathfrak{R}(w)>0$ and satisfies

$$
B(z, w)=\frac{\Gamma(z) \Gamma(w)}{\Gamma(z+w)}=B(w, z) .
$$

From the integral representations (8) and (9), one can not apparently see any message about the monotonicity and convexity of the Catalan-Qi function $C(a, b ; x)$ in $x \in[0, \infty)$.

As showed by (1), the Catalan numbers $C_{n}$ have a generating function $\frac{2}{1+\sqrt{1-4 x}}$. What is the generating function of the Catalan-Qi numbers $C(a, b ; n)$ ?

The aim of this paper is to supply answers to the above problems and others. 


\section{A new expression of the Catalan numbers}

In order to establish a new expression for the Catalan numbers $C_{n}$, we need the following lemma which was summarized up in the papers Qi (2015c, Section 2.2, p. 849), Qi (2016, p. 94), and Wei and Qi (2015, Lemma 2.1) from Bourbaki (2004, p. 40, Exercise 5).

Lemma 1 Let $u(x)$ and $v(x) \neq 0$ be differentiable functions, let $U_{(n+1) \times 1}(x)$ be an $(n+1) \times 1$ matrix whose elements $u_{k, 1}(x)=u^{(k-1)}(x)$ for $1 \leq k \leq n+1$, let $V_{(n+1) \times n}(x)$ be an $(n+1) \times n$ matrix whose elements

$$
v_{i, j}(x)= \begin{cases}\left(\begin{array}{l}
i-1 \\
j-1
\end{array}\right) v^{(i-j)}(x), & i-j \geq 0 \\
0, & i-j<0\end{cases}
$$

for $1 \leq i \leq n+1$ and $1 \leq j \leq n$, and let $\left|W_{(n+1) \times(n+1)}(x)\right|$ denote the determinant of the $(n+1) \times(n+1)$ matrix

$$
W_{(n+1) \times(n+1)}(x)=\left[U_{(n+1) \times 1}(x) V_{(n+1) \times n}(x)\right] .
$$

Then the nth derivative of the ratio $\frac{u(x)}{v(x)}$ can be computed by

$$
\frac{\mathrm{d}^{n}}{\mathrm{~d} x^{n}}\left[\frac{u(x)}{v(x)}\right]=(-1)^{n} \frac{\left|W_{(n+1) \times(n+1)}(x)\right|}{v^{n+1}(x)}
$$

Making use of the formula (13) in Lemma 1, we can obtain the following new expression for the Catalan numbers $C_{n}$.

Theorem 1 For $n \in \mathbb{N}$, the nth derivative of the generating function of the Catalan numbers $C_{n}$ can be expressed as

$$
\frac{\mathrm{d}^{n}}{\mathrm{~d} x^{n}}\left(\frac{1-\sqrt{1-4 x}}{2 x}\right)=\frac{(-1)^{n+1}}{2 x^{n+1}} \sum_{k=0}^{n} 4^{k}\left\langle\frac{1}{2}\right\rangle_{k} x^{k}(1-4 x)^{1 / 2-k}
$$

and the Catalan numbers $C_{n}$ can be represented as

$$
C_{n}=\frac{4^{n}}{(n+1) !}\left(\frac{1}{2}\right)_{n}
$$

where $\langle x\rangle_{n}$ is the falling factorial defined by

$$
\langle x\rangle_{n}=\prod_{k=0}^{n-1}(x-k)= \begin{cases}x(x-1) \cdots(x-n+1), & n \geq 1, \\ 1, & n=0\end{cases}
$$

and $(x)_{n}$ is the rising factorial defined by (3). 
Proof Let $u(x)=1-\sqrt{1-4 x}$ and $v(x)=x$. Since

$$
u^{(k)}(x)=(-1)^{k+1} 4^{k}\left\langle\frac{1}{2}\right\rangle_{k}(1-4 x)^{1 / 2-k} \rightarrow(-1)^{k+1} 4^{k}\left\langle\frac{1}{2}\right\rangle_{k}
$$

for $k \in \mathbb{N}$ as $x \rightarrow 0$, making use of the formula (13) yields

$$
\begin{aligned}
& \frac{\mathrm{d}^{n}}{\mathrm{~d} x^{n}}\left(\frac{1-\sqrt{1-4 x}}{2 x}\right) \\
& =\frac{(-1)^{n}}{2 x^{n+1}}\left(\begin{array}{cccccccc}
u(x) & x & 0 & 0 & \cdots & 0 & 0 & 0 \\
u^{\prime}(x) & \left(\begin{array}{l}
1 \\
0
\end{array}\right) & x & 0 & \cdots & 0 & 0 & 0 \\
u^{\prime \prime}(x) & 0 & \left(\begin{array}{l}
2 \\
1
\end{array}\right) & x & \cdots & 0 & 0 & 0 \\
u^{\prime \prime \prime}(x) & 0 & 0 & \left(\begin{array}{l}
3 \\
2
\end{array}\right) & \cdots & 0 & 0 & 0 \\
\vdots & \vdots & \vdots & \vdots & \ddots & \vdots & \vdots & \vdots \\
u^{(n-2)}(x) & 0 & 0 & 0 & \cdots & \left(\begin{array}{c}
n-2 \\
n-3
\end{array}\right) & x & 0 \\
u^{(n-1)}(x) & 0 & 0 & 0 & \cdots & 0 & \left(\begin{array}{c}
n-1 \\
n-2
\end{array}\right) & x \\
u^{(n)}(x) & 0 & 0 & 0 & \cdots & 0 & \left(\begin{array}{c}
n \\
n-1
\end{array}\right)
\end{array} \mid\right. \\
& =\frac{(-1)^{n}}{2 x^{n+1}}\left|\begin{array}{cccccccc}
u(x) & x & 0 & 0 & \cdots & 0 & 0 & 0 \\
u^{\prime}(x) & 1 & x & 0 & \cdots & 0 & 0 & 0 \\
u^{\prime \prime}(x) & 0 & 2 & x & \cdots & 0 & 0 & 0 \\
u^{\prime \prime \prime}(x) & 0 & 0 & 3 & \cdots & 0 & 0 & 0 \\
\vdots & \vdots & \vdots & \vdots & \ddots & \vdots & \vdots & \vdots \\
u^{(n-2)}(x) & 0 & 0 & 0 & \cdots & n-2 & x & 0 \\
u^{(n-1)}(x) & 0 & 0 & 0 & \cdots & 0 & n-1 & x \\
u^{(n)}(x) & 0 & 0 & 0 & \cdots & 0 & 0 & n
\end{array}\right|=\frac{(-1)^{n}}{2 x^{n+1}} \\
& \times\left|\begin{array}{cccccccc}
u(x) & x & 0 & 0 & \cdots & 0 & 0 & 0 \\
u^{\prime}(x)-\frac{u(x)}{x} & 0 & x & 0 & \cdots & 0 & 0 & 0 \\
u^{\prime \prime}(x)-\frac{2}{x}\left[u^{\prime}(x)-\frac{u(x)}{x}\right] & 0 & 0 & x & \cdots & 0 & 0 & 0 \\
u^{\prime \prime \prime}(x)-\frac{3}{x}\left\{u^{\prime \prime}(x)-\frac{2}{x}\left[u^{\prime}(x)-\frac{u(x)}{x}\right]\right\} & 0 & 0 & 0 & \cdots & 0 & 0 & 0 \\
\vdots & \vdots & \vdots & \vdots & \ddots & \vdots & \vdots & \vdots \\
u^{(n-2)}(x)-\sum_{k=1}^{n-2}(-1)^{k} \frac{(n-2) !}{(n-k-2) !} \frac{u^{(n-k-2)}(x)}{x^{k}} & 0 & 0 & 0 & \ldots & 0 & x & 0 \\
u^{(n-1)}(x)-\sum_{k=1}^{n-1}(-1)^{k} \frac{(n-1) !}{(n-k-1) !} \frac{u^{(n-k-1)}(x)}{x^{k}} & 0 & 0 & 0 & \ldots & 0 & 0 & x \\
u^{(n)}(x)-\sum_{k=1}^{n}(-1)^{k} \frac{n !}{(n-k) !} \frac{u^{(n-k)}(x)}{x^{k}} & 0 & 0 & 0 & \ldots & 0 & 0 & 0
\end{array}\right| \\
& =\frac{1}{2 x^{n+1}}\left[u^{(n)}(x)-\sum_{k=1}^{n}(-1)^{k} \frac{n !}{(n-k) !} \frac{u^{(n-k)}(x)}{x^{k}}\right]\left|\begin{array}{ccccccc}
x & 0 & 0 & \cdots & 0 & 0 & 0 \\
0 & x & 0 & \cdots & 0 & 0 & 0 \\
0 & 0 & x & \cdots & 0 & 0 & 0 \\
0 & 0 & 0 & \cdots & 0 & 0 & 0 \\
\vdots & \vdots & \vdots & \ddots & \vdots & \vdots & \vdots \\
0 & 0 & 0 & \cdots & 0 & x & 0 \\
0 & 0 & 0 & \cdots & 0 & 0 & x
\end{array}\right|
\end{aligned}
$$




$$
\begin{aligned}
& =\frac{1}{2 x}\left[u^{(n)}(x)-\sum_{k=1}^{n}(-1)^{k} \frac{n !}{(n-k) !} \frac{u^{(n-k)}(x)}{x^{k}}\right] \\
& =\frac{1}{2} \sum_{k=0}^{n}(-1)^{k} \frac{n !}{(n-k) !} \frac{u^{(n-k)}(x)}{x^{k+1}} \\
& =\frac{1}{2 x^{n+1}} \sum_{k=0}^{n}(-1)^{k} \frac{n !}{(n-k) !} x^{n-k} u^{(n-k)}(x) \\
& =(-1)^{n} \frac{1}{2 x^{n+1}} \sum_{k=0}^{n}(-1)^{k} \frac{n !}{k !} x^{k} u^{(k)}(x) \\
& \rightarrow \frac{(-1)^{n}}{2(n+1) !} \sum_{k=0}^{n}(-1)^{k} \frac{n !}{k !} \lim _{x \rightarrow 0}\left[x^{k} u^{(k)}(x)\right]^{(n+1)} \\
& =\frac{(-1)^{n}}{2(n+1) !} \sum_{k=0}^{n}(-1)^{k} \frac{n !}{k !} \lim _{x \rightarrow 0} \sum_{\ell=0}^{n+1}\left(\begin{array}{c}
n+1 \\
\ell
\end{array}\right)\left(x^{k}\right)^{(\ell)} u^{(n-\ell+k)}(x) \\
& =\frac{(-1)^{n}}{2(n+1) !} \sum_{k=0}^{n}(-1)^{k} \frac{n !}{k !}\left(\begin{array}{c}
n+1 \\
k
\end{array}\right) k ! u^{(n)}(0) \\
& =\frac{(-1)^{n}}{2(n+1)} \sum_{k=0}^{n}(-1)^{k}\left(\begin{array}{c}
n+1 \\
k
\end{array}\right) u^{(n)}(0) \\
& =\frac{(-1)^{n}}{2(n+1)}(-1)^{n+1} 4^{n}\left\langle\frac{1}{2}\right\rangle \sum_{n=0}^{n}(-1)^{k}\left(\begin{array}{c}
n+1 \\
k
\end{array}\right) \\
& =-\frac{4^{n}}{2(n+1)}\left\langle\frac{1}{2}\right\rangle \sum_{n=0}^{n}(-1)^{k}\left(\begin{array}{c}
n+1 \\
k
\end{array}\right) \\
& =(-1)^{n+1} \frac{4^{n}}{2(n+1)}\left\langle\frac{1}{2}\right\rangle_{n} \\
& =\frac{4^{n}}{n+1}\left\langle-\frac{1}{2}\right\rangle_{n}=\frac{4^{n}}{n+1}\left(\frac{1}{2}\right)_{n}
\end{aligned}
$$

as $x \rightarrow 0$. By virtue of the second function in the Eq. (1), we see that

$$
\begin{aligned}
C_{n} & =\frac{1}{n !} \lim _{x \rightarrow 0} \frac{\mathrm{d}^{n}}{\mathrm{~d} x^{n}}\left(\frac{1-\sqrt{1-4 x}}{2 x}\right) \\
& =(-1)^{n} \frac{4^{n}}{(n+1) !}\left\langle-\frac{1}{2}\right\rangle_{n}=\frac{4^{n}}{(n+1) !}\left(\frac{1}{2}\right)_{n} .
\end{aligned}
$$

The proof of Theorem 1 is complete.

\section{Asymptotic expansions of the Catalan-Qi function $C(\boldsymbol{a}, \boldsymbol{b} ; \boldsymbol{x})$}

We first derive two asymptotic expansions of the Catalan-Qi function $C(a, b ; x)$. Consequently, from these two asymptotic expansions, we deduce a general expression for (5) and an asymptotic expansion of the ratio $\frac{\Gamma(a)}{\Gamma(b)}$ for $a, b>0$.

Theorem 2 Let $B_{k}^{(\sigma)}(x)$ denote the generalized Bernoulli polynomials defined by

$$
e^{x z}\left(\frac{z}{e^{z}-1}\right)^{\sigma}=\sum_{k=0}^{\infty} \frac{B_{k}^{(\sigma)}(x)}{k !} z^{k}, \quad \sigma \in \mathbb{C}, \quad|z|<2 \pi .
$$


For $b>a>0$, the Catalan-Qi function $C(a, b ; x)$ has the asymptotic expansion

$$
C(a, b ; x) \sim \frac{\Gamma(b)}{\Gamma(a)}\left(\frac{b}{a}\right)^{x} \sum_{k=0}^{\infty}(-1)^{k} \frac{B_{k}^{(a-b+1)}(a)}{k !} \frac{\Gamma(b-a+k)}{\Gamma(b-a)} \frac{1}{x^{k+b-a}}
$$

as $x \rightarrow \infty$. Consequently, the Catalan function $C_{x}$ has the asymptotic expansion

$$
C_{x}=C\left(\frac{1}{2}, 2 ; x\right) \sim \frac{4^{x}}{\sqrt{\pi}} \sum_{k=0}^{\infty}(-1)^{k} \frac{B_{k}^{(-1 / 2)}(1 / 2)}{k !} \frac{\Gamma(k+3 / 2)}{\Gamma(3 / 2)} \frac{1}{x^{k+3 / 2}}
$$

as $x \rightarrow \infty$.

Proof In Temme (1996, p. 67), it was listed that, under the condition $\Re(b-a)>0$,

$$
\frac{\Gamma(z+a)}{\Gamma(z+b)} \sim z^{a-b} \sum_{k=0}^{\infty}(-1)^{k} \frac{B_{k}^{(a-b+1)}(a)}{k !} \frac{\Gamma(b-a+k)}{\Gamma(b-a)} \frac{1}{z^{k}} \quad \text { as } z \rightarrow \infty
$$

in the sector $|\arg z|<\pi$, where the generalized Bernoulli polynomials $B_{k}^{(\sigma)}(x)$ are defined by (14) in Temme (1996, p. 4). Consequently, the function $C(a, b ; x)$ has the asymptotic expansion (15) under the condition $b>a>0$ as $x \rightarrow \infty$. In particular, when taking $a=\frac{1}{2}$ and $b=2$ in (15), we obtain the asymptotic expansion (16). Theorem 2 is thus proved.

Remark 1 In Qi (2015a), there are another two asymptotic expansions for $C_{n}$ and $C_{x}$, which were established by virtue of the integral representations (8) and (7) for $a=\frac{1}{2}$ and $b=2$.

Remark 2 The asymptotic expansion (16) is a general expression of the asymptotic expansion (5). Hence, the asymptotic expansion (15) is a generalization of (5).

Theorem 3 Let $B_{i}$ denote the Bernoulli numbers defined by

$$
\frac{x}{e^{x}-1}=\sum_{i=0}^{\infty} B_{i} \frac{x^{i}}{i !}=1-\frac{x}{2}+\sum_{j=1}^{\infty} B_{2 j} \frac{x^{2 j}}{(2 j) !}, \quad|x|<2 \pi .
$$

Then the Catalan-Qi function $C(a, b ; x)$ has the exponential expansion

$$
\begin{aligned}
C(a, b ; x)= & \frac{\Gamma(b)}{\Gamma(a)}\left(\frac{b}{a}\right)^{x} \sqrt{\frac{x+b}{x+a}}[I(x+a, x+b)]^{a-b} \\
& \times \exp \left[\sum_{j=1}^{\infty} \frac{B_{2 j}}{2 j(2 j-1)}\left(\frac{1}{(x+a)^{2 j-1}}-\frac{1}{(x+b)^{2 j-1}}\right)\right], \quad a, b>0,
\end{aligned}
$$

where $I(\alpha, \beta)$ denotes the exponential mean defined by

$$
I(\alpha, \beta)=\frac{1}{e}\left(\frac{\beta^{\beta}}{\alpha^{\alpha}}\right)^{1 /(\beta-\alpha)}
$$


for $\alpha, \beta>0$ with $\alpha \neq \beta$. Consequently, we have

$$
\frac{\Gamma(a)}{\Gamma(b)}=\sqrt{\frac{b}{a}} \frac{a^{a}}{b^{b}} \exp \left[\sum_{j=1}^{\infty} \frac{B_{2 j}}{2 j(2 j-1)}\left(\frac{1}{a^{2 j-1}}-\frac{1}{b^{2 j-1}}\right)\right], \quad a, b>0 .
$$

Proof Making use of (17) in the integral representation (7) yields

$$
\begin{aligned}
C(a, b ; x)= & \frac{\Gamma(b)}{\Gamma(a)}\left(\frac{b}{a}\right)^{x} \frac{(x+a)^{x}}{(x+b)^{x+b-a}} \exp [b-a \\
& \left.+\int_{0}^{\infty} \frac{1}{t}\left(\frac{1}{e^{t}-1}-\frac{1}{t}+1-a\right)\left(e^{-a t}-e^{-b t}\right) e^{-x t} \mathrm{~d} t\right] \\
= & \frac{\Gamma(b)}{\Gamma(a)}\left(\frac{b}{a}\right)^{x} \frac{(x+a)^{x}}{(x+b)^{x+b-a}} \exp [b-a \\
& \left.+\int_{0}^{\infty} \frac{1}{t}\left(\frac{1}{2}-a+\sum_{j=1}^{\infty} B_{2 j} \frac{t^{2 j-1}}{(2 j) !}\right)\left(e^{-a t}-e^{-b t}\right) e^{-x t} \mathrm{~d} t\right] \\
= & \frac{\Gamma(b)}{\Gamma(a)}\left(\frac{b}{a}\right)^{x} \frac{(x+a)^{x}}{(x+b)^{x+b-a}} \exp \left[b-a+\left(\frac{1}{2}-a\right) \ln \frac{x+b}{x+a}\right. \\
& \left.+\sum_{j=1}^{\infty} \frac{B_{2 j}}{2 j(2 j-1)}\left(\frac{1}{(x+a)^{2 j-1}}-\frac{1}{(x+b)^{2 j-1}}\right)\right] \\
= & \frac{\Gamma(b)}{\Gamma(a)}\left(\frac{b}{a}\right)^{x} \frac{(x+a)^{x+a-1 / 2}}{(x+b)^{x+b-1 / 2}} e^{b-a} \\
& \times \exp \left[\sum_{j=1}^{\infty} \frac{B_{2 j}}{2 j(2 j-1)}\left(\frac{1}{(x+a)^{2 j-1}}-\frac{1}{(x+b)^{2 j-1}}\right)\right]
\end{aligned}
$$

which can be reformulated as the form (18).

The exponential expansion (20) follows from letting $x \rightarrow 0$ in (18) and rearranging. Theorem 3 is thus proved.

Remark 3 When taking $a=\frac{1}{2}$ and $b=2$, the asymptotic expansion (18) reduces to one of conclusions in Qi (2015a, Theorem 1.2).

Remark 4 For more information on the exponential mean $I(\alpha, \beta)$ in (19), please refer to the monograph (Bullen 2003) and the papers (Guo and Qi 2009, 2011).

\section{Integral representations and complete monotonicity of the Catalan-Qi function $C(a, b ; x)$}

Motivated by the first integral representations (8) and (9), we guess out the following integral representations for the Catalan-Qi function $C(a, b ; x)$.

Theorem 4 For $b>a>0$ and $x \geq 0$, the Catalan-Qi function $C(a, b ; x)$ has integral representations

$$
C(a, b ; x)=\left(\frac{a}{b}\right)^{b-1} \frac{1}{B(a, b-a)} \int_{0}^{b / a}\left(\frac{b}{a}-t\right)^{b-a-1} t^{x+a-1} \mathrm{~d} t
$$


and

$$
C(a, b ; x)=\left(\frac{a}{b}\right)^{a} \frac{1}{B(a, b-a)} \int_{0}^{\infty} \frac{t^{b-a-1}}{(t+a / b)^{x+b}} \mathrm{~d} t .
$$

Proof Straightforwardly computing and directly utilizing (11) and (12) acquire

$$
\begin{aligned}
\int_{0}^{\infty} \frac{t^{b-a-1}}{(t+a / b)^{x+b}} \mathrm{~d} t & =\left(\frac{b}{a}\right)^{x+b} \int_{0}^{\infty} \frac{t^{b-a-1}}{(1+b t / a)^{x+b}} \mathrm{~d} t \\
& =\left(\frac{b}{a}\right)^{x+b}\left(\frac{b}{a}\right)^{a-b} \int_{0}^{\infty} \frac{\left(\frac{b t}{a}\right)^{b-a-1}}{\left(1+\frac{b t}{a}\right)^{x+b}} \mathrm{~d}\left(\frac{b t}{a}\right) \\
& =\left(\frac{b}{a}\right)^{x+a} \int_{0}^{\infty} \frac{u^{b-a-1}}{(1+u)^{b-a+(x+a)}} \mathrm{d} u \\
& =\left(\frac{b}{a}\right)^{x+a} B(b-a, x+a) \\
& =\left(\frac{b}{a}\right)^{x+a} \frac{\Gamma(b-a) \Gamma(x+a)}{\Gamma(x+b)}
\end{aligned}
$$

The integral representation (21) is thus proved.

Similar to the above argument, by virtue of (11) and (12), we obtain

$$
\begin{aligned}
\int_{0}^{b / a}\left(\frac{b}{a}-t\right)^{b-a-1} t^{x+a-1} \mathrm{~d} t & =\left(\frac{b}{a}\right)^{x+b-1} \int_{0}^{1}(1-s)^{b-a-1} s^{x+a-1} \mathrm{~d} s \\
& =\left(\frac{b}{a}\right)^{x+b-1} B(b-a, x+a)=\left(\frac{b}{a}\right)^{x+b-1} \frac{\Gamma(b-a) \Gamma(x+a)}{\Gamma(x+b)} .
\end{aligned}
$$

Hence, the integral representation (22) follows readily. The proof of Theorem 4 is thus complete.

Remark 5 Letting $a=\frac{1}{2}, b=2$, and $x=n$ in (22) and (21) respectively reduce to the first integral representation in (9) and its equivalent form (8).

Remark 6 In https://en.wikipedia.org/wiki/Catalan_number, it was said that the integral representation (8) means that the Catalan numbers $C_{n}$ are a solution of the Hausdorff moment problem on the interval $[0,4]$ instead of $[0,1]$. Analogously, we guess that the integral representation (21) probably means that the Catalan-Qi numbers $C(a, b ; n)$ are a solution of the Hausdorff moment problem on the interval $\left[0, \frac{b}{a}\right]$ instead of $[0,1]$ and $[0,4]$.

Recall from Mitrinović et al. (1993, Chapter XIII), Schilling et al. (2012, Chapter 1), and Widder (1941, Chapter IV) that an infinitely differentiable function $f$ is said to be completely monotonic on an interval $I$ if it satisfies $0 \leq(-1)^{k} f^{(k)}(x)<\infty$ on $I$ for all $k \geq 0$. It is known (Widder 1941, p. 161, Theorem 12b) that a function $f$ is completely monotonic on $(0, \infty)$ if and only if it is a Laplace transform $f(t)=\int_{0}^{\infty} e^{-t s} \mathrm{~d} \mu(s)$ of a positive measure $\mu$ defined on $[0, \infty)$ such that the above integral converges on $(0, \infty)$. 
Theorem 5 For $b>a>0$, we have

$$
C(a, b ; x)=\frac{1}{B(a, b-a)}\left(\frac{b}{a}\right)^{x} \sum_{k=0}^{\infty}(-1)^{k} \frac{\langle b-a-1\rangle_{k}}{k !} \frac{1}{x+a+k},
$$

where

$$
\langle x\rangle_{n}=\prod_{k=0}^{n-1}(x-k)= \begin{cases}x(x-1) \cdots(x-n+1), & n \geq 1 \\ 1, & n=0\end{cases}
$$

is the falling factorial. Consequently, the function

$$
(-1)^{\lfloor b-a\rfloor}\left[\left(\frac{a}{b}\right)^{x} C(a, b ; x)-\frac{1}{B(a, b-a)} \sum_{k=0}^{N}(-1)^{k} \frac{\langle b-a-1\rangle_{k}}{k !} \frac{1}{x+a+k}\right]
$$

for $N \in\{0\} \cup \mathbb{N}$ and $b>a>0$ is completely monotonic in $x \in[0, \infty)$, where $\lfloor x\rfloor$ denotes the floor function whose value is the largest integer less than or equal to $x$.

Proof The integral representation (21) can be rearranged as

$$
\begin{aligned}
C(a, b ; x) & =\frac{1}{B(a, b-a)}\left(\frac{b}{a}\right)^{x-1} \int_{0}^{b / a}\left(1-\frac{a}{b} t\right)^{b-a-1}\left(\frac{a}{b} t\right)^{x+a-1} \mathrm{~d} t \\
& =\frac{1}{B(a, b-a)}\left(\frac{b}{a}\right)^{x} \int_{0}^{1}(1-s)^{b-a-1} s^{x+a-1} \mathrm{~d} s .
\end{aligned}
$$

Further utilizing the well-known power series expansion

$$
(1+x)^{\alpha}=\sum_{k=0}^{\infty}\langle\alpha\rangle_{k} \frac{x^{k}}{k !}, \quad|x|<1
$$

arrives at

$$
\begin{aligned}
C(a, b ; x) & =\frac{1}{B(a, b-a)}\left(\frac{b}{a}\right)^{x} \sum_{k=0}^{\infty} \frac{(-1)^{k}}{k !}\langle b-a-1\rangle_{k} \int_{0}^{1} s^{x+k+a-1} \mathrm{~d} s \\
& =\frac{1}{B(a, b-a)}\left(\frac{b}{a}\right)^{x} \sum_{k=0}^{\infty} \frac{(-1)^{k}}{k !}\langle b-a-1\rangle_{k} \frac{1}{x+a+k}
\end{aligned}
$$

which can be reformulated as (23).

Rewriting (23) as

$$
\begin{aligned}
& \left(\frac{a}{b}\right)^{x} C(a, b ; x)-\frac{1}{B(a, b-a)} \sum_{k=0}^{N}(-1)^{k} \frac{\langle b-a-1\rangle_{k}}{k !} \frac{1}{x+a+k} \\
& =\frac{1}{B(a, b-a)} \sum_{k=N+1}^{\infty}(-1)^{k} \frac{\langle b-a-1\rangle_{k}}{k !} \frac{1}{x+a+k} \\
& =(-1)^{\lfloor b-a\rfloor} \frac{1}{B(a, b-a)} \sum_{k=N+1}^{\infty}(-1)^{k-\lfloor b-a\rfloor} \frac{\langle b-a-1\rangle_{k}}{k !} \frac{1}{x+a+k},
\end{aligned}
$$


considering the non-negativity of $(-1)^{k-\lfloor b-a\rfloor}\langle b-a-1\rangle_{k}$, and employing the complete monotonicity of $\frac{1}{x+a+k}$ in $x \in[0, \infty)$ reveal the complete monotonicity of the function (24). The proof of Theorem 5 is complete.

Remark 7 When taking $a=\frac{1}{2}$ and $b=2$, Theorem 5 becomes a part of conclusions in Qi (2015a, Theorem 1.1).

\section{Logarithmically complete monotonicity of the Catalan-Qi function $C(a, b ; x)$}

An infinitely differentiable and positive function $f$ is said to be logarithmically completely monotonic on an interval $I$ if $0 \leq(-1)^{k}[\ln f(x)]^{(k)}<\infty$ hold on $I$ for all $k \in \mathbb{N}$. The inclusions

$$
\mathcal{L}[I] \subset \mathcal{C}[I] \quad \text { and } \quad \mathcal{S} \backslash\{0\} \subset \mathcal{L}[(0, \infty)]
$$

were discovered in Berg (2004), Guo and Qi (2010), Qi and Chen (2004), Qi and Guo (2004), where $\mathcal{L}[I], \mathcal{C}[I]$, and $\mathcal{S}$ denote respectively the set of all logarithmically completely monotonic functions on an interval $I$, the set of all completely monotonic functions on $I$, and the set of all Stieltjes transforms. See also the monograph Schilling et al. (2012) and plenty of references therein.

Recall from monographs Mitrinović et al. (1993, pp. 372-373) and Widder (1941, p. 108, Definition 4) that a sequence $\left\{\mu_{n}\right\}_{0 \leq n \leq \infty}$ is said to be completely monotonic if its elements are non-negative and its successive differences are alternatively nonnegative, that is,

$$
(-1)^{k} \Delta^{k} \mu_{n} \geq 0
$$

for $n, k \geq 0$, where

$$
\Delta^{k} \mu_{n}=\sum_{m=0}^{k}(-1)^{m}\left(\begin{array}{c}
k \\
m
\end{array}\right) \mu_{n+k-m} .
$$

Recall from Widder (1941, p. 163, Definition 14a) that a completely monotonic sequence $\left\{a_{n}\right\}_{n \geq 0}$ is minimal if it ceases to be completely monotonic when $a_{0}$ is decreased.

\section{Theorem 6 The function}

$$
\mathcal{C}^{ \pm 1}(a, b ; x)= \begin{cases}1, & x=0 \\ {[C(a, b ; x)]^{ \pm 1 / x},} & x>0\end{cases}
$$

is logarithmically completely monotonic on $(0, \infty)$ if and only if $a \gtrless b$. Consequently, the sequence

$$
\mathcal{C}_{n}= \begin{cases}1, & n=0 \\ \frac{1}{\sqrt[n]{C_{n}}}, & n \in \mathbb{N}\end{cases}
$$

is completely monotonic, minimal, and logarithmically convex. 
Proof In Qi and Li (2015, Theorem 1.1), it was proved that, when $a \gtrless b$, the function

$$
\left[h_{a, b ; c}(x)\right]^{ \pm 1}= \begin{cases}1, & x=0 \\ {\left[c \frac{\Gamma(x+a)}{\Gamma(x+b)}\right]^{ \pm 1 / x},} & x>0\end{cases}
$$

for $c>0$ is logarithmically completely monotonic on $[0, \infty)$ if and only if $c \gtreqless \frac{\Gamma(b)}{\Gamma(a)}$. It is easy to see that

$$
\mathcal{C}^{ \pm 1}(a, b ; x)=\left(\frac{b}{a}\right)^{ \pm 1}\left[h_{a, b ; \Gamma(b) / \Gamma(a)}(x)\right]^{ \pm 1}
$$

Therefore, the function $\mathcal{C}^{ \pm 1}(a, b ; x)$ is logarithmically completely monotonic on $[0, \infty)$ if and only if $a \gtrless b$. Consequently, the function $\mathcal{C}^{-1}\left(\frac{1}{2}, 2 ; x\right)$ is logarithmically completely monotonic, and then completely monotonic and logarithmically convex, on $[0, \infty)$. As a result, the complete monotonicity, minimality, and logarithmic convexity of the sequence (27) follows immediately from Widder (1941, p. 164, Theorem 14b) which reads that a necessary and sufficient condition that there should exist a completely monotonic function $f(x)$ in $0 \leq x<\infty$ such that $f(n)=a_{n}$ for $n \geq 0$ is that $\left\{a_{n}\right\}_{0}^{\infty}$ should be a minimal completely monotonic sequence. The proof of Theorem 6 is complete.

Remark 8 It is interesting that, since the function $h_{a, b ; c}(x)$ defined by (28) originates from the coding gain (see Lee and Tepedelenlioğlu 2011; Qi and Li 2015), Theorem 6 and its proof imply some connections and relations among the Catalan numbers, the coding gain, and the ratio of two gamma functions.

Theorem 7 Let $a, b>0$ and $x \geq 0$. Then

1. when $b>a$, the function $C(a, b ; x)$ is decreasing in $x \in\left[0, x_{0}\right)$, increasing in $x \in\left(x_{0}, \infty\right)$, and logarithmically convex in $x \in[0, \infty)$;

2. when $b<a$, the function $C(a, b ; x)$ is increasing in $x \in\left[0, x_{0}\right)$, decreasing in $x \in\left(x_{0}, \infty\right)$, and logarithmically concave in $x \in[0, \infty)$;

where $x_{0}$ is the unique zero of the equation

$$
\frac{\psi(x+b)-\psi(x+a)}{\ln b-\ln a}=1
$$

and satisfies $x_{0} \in\left(0, \frac{1}{2}\right)$. Consequently, the Catalan numbers $C_{n}$ for $n \in \mathbb{N}$ is strictly increasing and logarithmically convex.

Proof In Guo and Qi (2010, Theorem 1) closely-related references therein, it was proved that the function

$$
\theta_{\alpha}(x)=x^{\alpha}[\ln x-\psi(x)]
$$

is completely monotonic on $(0, \infty)$ if and only if $\alpha \leq 1$. This means that 


$$
\ln a-\psi(a) \lessgtr \ln b-\psi(b), \quad a \gtrless b,
$$

that is,

$$
\frac{\psi(b)-\psi(a)}{\ln b-\ln a}>1, \quad a \neq b .
$$

This can also be verified by virtue of the inequality

$$
\psi^{\prime}(x)>\frac{1}{x}+\frac{1}{2 x^{2}}>\frac{1}{x}, \quad x>0
$$

which is a special case of Guo and Qi (2010, Lemma 3), and by virtue of the equality

$$
\frac{\psi(b)-\psi(a)}{\ln b-\ln a}=\frac{\int_{a}^{b} \psi^{\prime}(x) \mathrm{d} x}{\int_{a}^{b} 1 / x \mathrm{~d} x} .
$$

Since the function $\psi(x+b)-\psi(x+a)$ is increasing (or decreasing, respectively) if and only if $b<a$ (or $b>a$, respectively) and

$$
\lim _{x \rightarrow \infty}[\psi(x+b)-\psi(x+a)]=0
$$

for all $a, b>0$, we obtain that for all $a, b>0$ with $a \neq b$ the function $\frac{\psi(x+b)-\psi(x+a)}{\ln b-\ln a}$ is strictly decreasing on $[0, \infty)$ and

$$
\lim _{x \rightarrow \infty} \frac{\psi(x+b)-\psi(x+a)}{\ln b-\ln a}=0 .
$$

It is clear that the first derivative

$$
\frac{\partial[\ln C(a, b ; x)]}{\partial x}=(\ln b-\ln a)-[\psi(x+b)-\psi(x+a)] \lesseqgtr 0
$$

if and only if

$$
\ln b-\ln a \lesseqgtr \psi(x+b)-\psi(x+a)
$$

which can be rewritten as

$$
\frac{\psi(x+b)-\psi(x+a)}{\ln b-\ln a} \gtreqless 1, \quad b>a
$$

and

$$
\frac{\psi(x+b)-\psi(x+a)}{\ln b-\ln a} \lesseqgtr 1, \quad b<a .
$$

As a result, considering (30) and (31), we see that the Catalan-Qi function $C(a, b ; x)$ for all $a, b>0$ with $a \neq b$ is not monotonic on $[0, \infty)$ and that 
1. when $b>a$, the function $C(a, b ; x)$ is decreasing in $x \in\left(0, x_{0}\right)$ and increasing in $x \in\left(x_{0}, \infty\right)$;

2. when $b<a$, the function $C(a, b ; x)$ is increasing in $x \in\left(0, x_{0}\right)$ and decreasing in $x \in\left(x_{0}, \infty\right)$;

where $x_{0}$ is the unique zero of the Eq. (29).

The Eq. (29) can be rearranged as

$$
\psi(x+b)-\psi(x+a)=\ln b-\ln a .
$$

Regarding $b$ as a variable and differentiating with respect to $b$ give

$$
\psi^{\prime}(x+b)=\frac{1}{b}=\frac{1}{(x+b)-x}
$$

which can be reformulated as

$$
x=(x+b)-\frac{1}{\psi^{\prime}(x+b)} \triangleq u-\frac{1}{\psi^{\prime}(u)},
$$

where $\lim _{u \rightarrow 0^{+}}\left[u-\frac{1}{\psi^{\prime}(u)}\right]=0$ and

$$
\frac{\mathrm{d}}{\mathrm{d} u}\left[u-\frac{1}{\psi^{\prime}(u)}\right]=1+\frac{\psi^{\prime \prime}(x)}{\left[\psi^{\prime}(x)\right]^{2}}=\frac{\left[\psi^{\prime}(x)\right]^{2}+\psi^{\prime \prime}(x)}{\left[\psi^{\prime}(x)\right]^{2}} .
$$

Employing the asymptotic expansion

$$
\psi^{\prime}(x)=\frac{1}{x}+\frac{1}{2 x^{2}}+\sum_{m=1}^{\infty} \frac{B_{2 m}}{x^{2 m+1}}
$$

in Abramowitz and Stegun (1972, p. 260, 6.4.11) yields

$$
u-\frac{1}{\psi^{\prime}(u)}=\frac{\frac{1}{2 u}+\sum_{m=1}^{\infty} \frac{B_{2 m}}{u^{2 m}}}{\frac{1}{u}+\frac{1}{2 u^{2}}+\sum_{m=1}^{\infty} \frac{B_{2 m}}{u^{2 m+1}}} \rightarrow \frac{1}{2}, \quad u \rightarrow \infty .
$$

Due to $\left[\psi^{\prime}(x)\right]^{2}+\psi^{\prime \prime}(x)>0$ on $(0, \infty)$, see Alzer (2004), Qi (2015b), Qi and Li (2015), Qi et al. (2013) and plenty of closely-related references therein, the function $u-\frac{1}{\psi^{\prime}(u)}$ is strictly increasing, and so

$$
0<u-\frac{1}{\psi^{\prime}(u)}<\frac{1}{2}
$$

on $(0, \infty)$. Accordingly, the unique zero $x_{0}$ of the Eq. (29) belongs to $\left(0, \frac{1}{2}\right)$.

It is immediate that

$$
\frac{\partial^{2}[\ln C(a, b ; x)]}{\partial x^{2}}=\psi^{\prime}(x+a)-\psi^{\prime}(x+b) .
$$

Since the tri-gamma function $\psi^{\prime}(x)$ is completely monotonic on $(0, \infty)$, inequalities

$$
(-1)^{k+1} \frac{\partial^{k+1}[\ln C(a, b ; x)]}{\partial x^{k+1}}=\psi^{(k)}(x+a)-\psi^{(k)}(x+b) \lessgtr 0
$$


for $k \in \mathbb{N}$ hold if and only if $b \lessgtr a$. The proof of Theorem 7 is complete.

Remark 9 From Theorem 7, we can derive that, for $b>a>0$,

$$
\frac{\Gamma(x+a)}{\Gamma(x+b)} \lessgtr \frac{\Gamma(a)}{\Gamma(b)}\left(\frac{a}{b}\right)^{x}, \quad 0<x \lessgtr 1 .
$$

In other words,

$$
0<C(a, b ; x) \lessgtr 1, \quad 0<x \lessgtr 1, \quad b>a>0 .
$$

Theorem 8 For $b>a>0$, the function

$$
\left(\frac{a}{b}\right)^{x} C(a, b ; x)
$$

is logarithmically completely monotonic on $[0, \infty)$.

Proof By (6), it follows that

$$
\left(\frac{a}{b}\right)^{x} C(a, b ; x)=\frac{\Gamma(b)}{\Gamma(a)} \frac{\Gamma(x+a)}{\Gamma(x+b)}
$$

which can be straightforwardly verified to be a logarithmically completely monotonic function on $[0, \infty)$. By the first inclusion in (26), we obtain the required complete monotonicity of the function (32).

Remark 10 The integral representation (22) can be rewritten as

$$
C(a, b ; x)=\frac{1}{B(a, b-a)}\left(\frac{b}{a}\right)^{x+b-a} \int_{0}^{\infty} \frac{t^{b-a-1}}{(b t / a+1)^{x+b}} \mathrm{~d} t
$$

for $b>a>0$ and $x \geq 0$. This formula and both of the integral representations (10) and (25) all mean that the function (32) for $b>a>0$ is completely monotonic on $[0, \infty)$. This conclusion is weaker than Theorem 8 .

Theorem 9 For $b>a>0$, the function

$$
\left(\frac{a}{b}\right)^{x} \frac{(x+b)^{x+b-a}}{(x+a)^{x}} C(a, b ; x)
$$

is logarithmically completely monotonic on $[0, \infty)$.

Proof This follows from the integral representation (7).

Remark 11 Theorems 8 and 9 imply that the sequences

$$
\left\{\frac{C_{n}}{4^{n}}\right\}_{n \geq 0} \text { and }\left\{\frac{(n+2)^{n+3 / 2}}{(n+1 / 2)^{n}} \frac{C_{n}}{4^{n}}\right\}_{n \geq 0}
$$

are logarithmically completely monotonic and minimal, which have been concluded in Qi (2015a, Theorems 1.1 and 1.2). 


\section{A generating function of the Catalan-Qi sequence $C(a, b ; n)$}

In this section, we discover that ${ }_{2} F_{1}\left(a, 1 ; b ; \frac{b t}{a}\right)$ is a generating function of the CatalanQi numbers $C(a, b ; n)$.

Theorem 10 For $a, b>0$ and $n \geq 0$, the Catalan-Qi numbers $C(a, b ; n)$ can be generated by

$$
{ }_{2} F_{1}\left(a, 1 ; b ; \frac{b t}{a}\right)=\sum_{n=0}^{\infty} C(a, b ; n) t^{n}
$$

and, conversely, satisfy

$$
C(a, b ; n)=(-1)^{n} \sum_{k=0}^{n}(-1)^{k}\left(\begin{array}{l}
n \\
k
\end{array}\right){ }_{2} F_{1}\left(a,-k ; b ;-\frac{b}{a}\right) .
$$

Proof Using the relation $(z)_{n} \Gamma(z)=\Gamma(z+n)$ for $n \geq 0$, we have

$$
C(a, b ; n)=\left(\frac{b}{a}\right)^{n} \frac{(a)_{n}}{(b)_{n}}, \quad a, b>0, \quad n \geq 0 .
$$

As a result, we obtain

$$
\sum_{n=0}^{\infty} C(a, b ; n) t^{n}=\sum_{n=0}^{\infty} \frac{(a)_{n}(1)_{n}}{(b)_{n}} \frac{(b t / a)^{n}}{n !}={ }_{2} F_{1}\left(a, 1 ; b ; \frac{b t}{a}\right), \quad a, b>0 .
$$

Using the relation $(-n)_{n+i}=0$ for $i \in \mathbb{N}$, which can be derived from (4), we obtain

$$
{ }_{2} F_{1}\left(a,-n ; b ;-\frac{b}{a}\right)=\sum_{r=0}^{n} \frac{(-1)^{r}(-n)_{r}}{r !} C(a, b ; r) .
$$

Further using the relation

$$
(-1)^{r}(-n)_{r}=(n-r+1)_{r}=\frac{\Gamma(n+1)}{\Gamma(n-r+1)}=\frac{n !}{(n-r) !},
$$

we acquire

$$
{ }_{2} F_{1}\left(a,-n ; b ;-\frac{b}{a}\right)=\sum_{r=0}^{n}\left(\begin{array}{l}
n \\
r
\end{array}\right) C(a, b ; r) .
$$

The formula (Graham et al. 1994, p. 192, (5.48)) reads that

$$
g(k)=\sum_{\ell}\left(\begin{array}{l}
k \\
\ell
\end{array}\right)(-1)^{\ell} f(\ell) \text { if and only if } f(k)=\sum_{\ell}\left(\begin{array}{l}
k \\
\ell
\end{array}\right)(-1)^{\ell} g(\ell) .
$$

Hence, the inversion of the relation (35) gives us the relation (34). The proof of Theorem 10 is complete. 
Remark 12 (An alternative proof of (33) for $b>1$ ) In Abramowitz and Stegun (1972, p. $558,15.3 .1)$, it is collected that

$$
{ }_{2} F_{1}(a, b ; c ; z)=\frac{1}{B(b, c-b)} \int_{0}^{1} \frac{t^{b-1}(1-t)^{c-b-1}}{(1-t z)^{a}} \mathrm{~d} t, \quad \mathfrak{R}(c)>\mathfrak{R}(b)>0 .
$$

In order to prove the Eq. (33), it is sufficient to show

$$
\lim _{t \rightarrow 0} \frac{\mathrm{d}^{n}}{\mathrm{~d} t^{n}}\left[{ }_{2} F_{1}\left(a, 1 ; b ; \frac{b t}{a}\right)\right]=n ! C(a, b ; n)
$$

In fact, a straightforward calculation reveals

$$
\begin{aligned}
\lim _{z \rightarrow 0} & \frac{\mathrm{d}^{n}}{\mathrm{~d} z^{n}}\left[\frac{1}{B(1, b-1)} \int_{0}^{1} \frac{(1-t)^{b-2}}{(1-b t z / a)^{a}} \mathrm{~d} t\right] \\
= & (b-1) a^{a} \lim _{z \rightarrow 0} \frac{\mathrm{d}^{n}}{\mathrm{~d} z^{n}} \int_{0}^{1} \frac{(1-t)^{b-2}}{(a-b t z)^{a}} \mathrm{~d} t \\
& =(b-1) a^{a} b^{n} \frac{\Gamma(n+a)}{\Gamma(a)} \lim _{z \rightarrow 0} \int_{0}^{1} \frac{t^{n}(1-t)^{b-2}}{(a-b t z)^{a+n}} \mathrm{~d} t \\
& =\frac{(b-1) a^{a} b^{n}}{a^{a+n}} \frac{\Gamma(n+a)}{\Gamma(a)} \int_{0}^{1} t^{n}(1-t)^{b-2} \mathrm{~d} t \\
& =\frac{(b-1) b^{n}}{\Gamma(n+a)} \frac{\Gamma(b-1) \Gamma(n+1)}{\Gamma(a)} \\
& =n ! \frac{a^{n}}{a^{n}} \frac{\Gamma(n+a)}{\Gamma(a)} \frac{\Gamma(b)}{\Gamma(n+b)} \\
& =n ! C(a, b ; n)
\end{aligned}
$$

for $b>1$. This gives an alternative proof of (33) for $b>1$.

Remark 13 Combining (2) and (34) brings out

$$
{ }_{2} F_{1}(1-n,-n ; 2 ; 1)=(-1)^{n} \sum_{k=0}^{n}(-1)^{k}\left(\begin{array}{l}
n \\
k
\end{array}\right){ }_{2} F_{1}\left(\frac{1}{2},-k ; 2 ;-4\right) .
$$

\section{A double inequality of the Catalan-Qi function $C(a, b ; x)$}

Finally we present a double inequality of the Catalan-Qi function $C(a, b ; x)$.

Theorem 11 Let $B_{i}$ for $i \in \mathbb{N}$ be the Bernoulli numbers defined by (17) and let I be the exponential mean defined by (19). Then the Catalan-Qi function $C(a, b ; x)$ satisfies the double inequality

$$
\begin{aligned}
\exp & {\left[\sum_{j=1}^{2 m} \frac{B_{2 j}}{2 j(2 j-1)}\left(\frac{1}{(x+a)^{2 j-1}}-\frac{1}{(x+b)^{2 j-1}}\right)\right] } \\
< & \frac{\Gamma(a)}{\Gamma(b)}\left(\frac{a}{b}\right)^{x} \sqrt{\frac{x+a}{x+b}} \frac{C(a, b ; x)}{[I(x+a, x+b)]^{a-b}} \\
& <\exp \left[\sum_{j=1}^{2 m-1} \frac{B_{2 j}}{2 j(2 j-1)}\left(\frac{1}{(x+a)^{2 j-1}}-\frac{1}{(x+b)^{2 j-1}}\right)\right] .
\end{aligned}
$$


Consequently, we have

$$
\begin{gathered}
\sqrt{\frac{b}{a}}[I(a, b)]^{a-b} \exp \left[\sum_{j=1}^{2 m} \frac{B_{2 j}}{2 j(2 j-1)}\left(\frac{1}{a^{2 j-1}}-\frac{1}{b^{2 j-1}}\right)\right]<\frac{\Gamma(a)}{\Gamma(b)} \\
<\sqrt{\frac{b}{a}}[I(a, b)]^{a-b} \exp \left[\sum_{j=1}^{2 m-1} \frac{B_{2 j}}{2 j(2 j-1)}\left(\frac{1}{a^{2 j-1}}-\frac{1}{b^{2 j-1}}\right)\right] .
\end{gathered}
$$

Proof In Koumandos (2006, Theorem 3), it was obtained that

$$
1-\frac{x}{2}+\sum_{j=1}^{2 m} \frac{B_{2 j}}{(2 j) !} x^{2 j}<\frac{x}{e^{x}-1}<1-\frac{x}{2}+\sum_{j=1}^{2 m-1} \frac{B_{2 j}}{(2 j) !} x^{2 j}
$$

for $m \in \mathbb{N}$ and $x>0$. Substituting this double inequality into the integral representation (7) and straightforward computing lead to the double inequality (36).

The double inequality (37) follows from letting $x \rightarrow 0$ in (36) and simplifying. The proof of Theorem 11 is complete.

Remark 14 The double inequality (36) generalizes a double inequality in Qi (2015a, Theorem 1.2).

\section{Conclusions}

The main conclusions of this paper are stated in Theorems 1, 2, 3, 4, 5, 6, 7, 8, 9, 10, and 11. Concretely speaking, a new expression, several asymptotic expansions, several integral representations, logarithmic convexity, complete monotonicity, minimality, logarithmically complete monotonicity, a generating function, and several inequalities of the Catalan numbers, the Catalan function, and the Catalan-Qi function are presented and an exponential expansion and a double inequality for the ratio of two gamma functions are derived. These conclusions generalize and extend some known results. More importantly, these conclusions provide new viewpoints of understanding and supply new methods of investigating the Catalan numbers in combinatorics and number theory. Moreover, these conclusions connect the Catalan numbers with the ratios of two gamma functions in the theory of special functions. In other words, the main conclusions in this paper will deepen and promote the study of the Catalan numbers and related concepts in combinatorics and number theory.

Remark 15 This paper is a companion of the articles Liu et al. (2015), Mahmoud and Qi (2016), Qi (2015a, d, e), Qi and Guo (2016a, b), Qi et al. (2015b, c, d, e), Shi et al. (2015) and a revised version of the preprint Qi et al. (2015a). 
${ }^{3}$ Department of Mathematics, Faculty of Science, Mansoura University, Mansoura 35516, Egypt. ${ }^{4}$ Department of Mathematics, School of Science, Tianjin Polytechnic University, Tianjin City 300387, China.

\section{Acknowledgements}

The authors appreciate anonymous referees for their careful corrections to and valuable comments on the original version of this paper.

\section{Competing interests}

The authors declare that they have no competing interests.

Received: 2 May 2016 Accepted: 7 July 2016

Published online: 19 July 2016

\section{References}

Abramowitz, M, Stegun IA (ed) (1972) Handbook of mathematical functions with formulas, graphs, and mathematical tables. National Bureau of Standards, Applied Mathematics Series 55, 10th printing, Washington

Alzer H (2004) Sharp inequalities for the digamma and polygamma functions. Forum Math 16(2):181-221. doi:10.1515/ form.2004.009

Berg C (2004) Integral representation of some functions related to the gamma function. Mediterr J Math 1(4):433-439. doi:10.1007/s00009-004-0022-6

Bourbaki N (2004) Functions of a real variable, elementary theory, translated from the 1976 French original by Philip Spain, Elements of mathematics (Berlin). Springer, Berlin. doi:10.1007/978-3-642-59315-4

Bullen PS (2003) Handbook of means and their inequalities, mathematics and its applications. Kluwer, Dordrecht. doi:10.1007/978-94-017-0399-4

Graham RL, Knuth DE, Patashnik O (1994) Concrete mathematics - a foundation for computer science, 2nd edn. Addison-Wesley Publishing Company, Reading

Guo B-N, Qi F (2010) A property of logarithmically absolutely monotonic functions and the logarithmically complete monotonicity of a power-exponential function. Politehn Univ Bucharest Sci Bull Ser A Appl Math Phys 72(2):21-30

Guo B-N, Qi F (2009) A simple proof of logarithmic convexity of extended mean values. Numer Algorithms 52(1):89-92. doi:10.1007/s11075-008-9259-7

Guo B-N, Qi F (2011) The function $\left(b^{x}-a^{x}\right) / x$ : logarithmic convexity and applications to extended mean values. Filomat 25(4):63-73. doi:10.2298/FIL1104063G

Guo B-N, Qi F (2010) Two new proofs of the complete monotonicity of a function involving the psi function. Bull Korean Math Soc 47(1):103-111. doi:10.4134/bkms.2010.47.1.103

Koshy T (2009) Catalan numbers with applications. Oxford University Press, Oxford

Koumandos S (2006) Remarks on some completely monotonic functions. J Math Anal Appl 324(2):1458-1461. doi:10.1016/j.jmaa.2005.12.017

Lee J, Tepedelenlioğlu C (2011) Space-time coding over fading channels with stable noise. IEEE Trans Veh Technol 60(7):3169-3177. doi:10.1109/TVT.2011.2160411

Liu F-F, Shi X-T, Qi F (2015) A logarithmically completely monotonic function involving the gamma function and originating from the Catalan numbers and function. Glob J Math Anal 3(4):140-144. doi:10.14419/gjma.v3i4.5187

Mahmoud M, Qi F (2016) Three identities of the Catalan-Qi numbers. Mathematics 4(2):7. doi:10.3390/math4020035

Mitrinović DS, Pečarić JE, Fink AM (1993) Classical and new inequalities in analysis. Kluwer, Dordrecht. doi:10.1007/978-94-017-1043-5

Nkwanta A, Tefera A (2013) Curious relations and identities involving the Catalan generating function and numbers. J Integer Seq 16(9), Article 13.9 .5

Qi F (2015a) Asymptotic expansions, complete monotonicity, and inequalities of the Catalan numbers. ResearchGate technical report. doi:10.13140/RG.2.1.4371.6321

Qi F (2015b) Complete monotonicity of a function involving the tri- and tetra-gamma functions. Proc Jangjeon Math Soc 18(2):253-264. doi:10.17777/pjms.2015.18.2.253

Qi F (2015c) Derivatives of tangent function and tangent numbers. Appl Math Comput 268:844-858. doi:10.1016/j. amc.2015.06.123

Qi F (2015d) Some properties and generalizations of the Catalan, Fuss, and Fuss-Catalan numbers. ResearchGate Res. doi:10.13140/RG.2.1.1778.3128

Qi F (2015e) Two product representations and several properties of the Fuss-Catalan numbers. ResearchGate Res. doi:10.13140/RG.2.1.1655.6004

Qi F, Chapman RJ (2016) Two closed forms for the Bernoulli polynomials. J Number Theory 159:89-100. doi:10.1016/j. jnt.2015.07.021

Qi F, Chen C-P (2004) A complete monotonicity property of the gamma function. J Math Anal Appl 296:603-607. doi:10.1016/j.jmaa.2004.04.026

Qi F, Guo B-N (2004) Complete monotonicities of functions involving the gamma and digamma functions. RGMIA Res Rep Coll 7(1):63-72. http://rgmia.org/v7n1.php

Qi F, Guo B-N (2016a) Logarithmically complete monotonicity of a function related to the Catalan-Qi function. Acta Univ Sapientiae Math 8(1):93-102. doi:10.1515/ausm-2016-0006

Qi F, Guo B-N (2016b) Logarithmically complete monotonicity of Catalan-Qi function related to Catalan numbers. Cogent Math 3:1179379. doi:10.1080/23311835.2016.1179379

Qi F, Li W-H (2015) A logarithmically completely monotonic function involving the ratio of gamma functions. J Appl Anal Comput 5(4):626-634. doi:10.11948/2015049 
Qi F, Luo Q-M, Guo B-N (2013) Complete monotonicity of a function involving the divided difference of digamma functions. Sci China Math 56(11):2315-2325. doi:10.1007/s11425-012-4562-0

Qi F, Mahmoud M, Shi X-T, Liu F-F (2015a) Some properties of the Catalan-Qi function related to the Catalan numbers. ResearchGate Technical report. doi:10.13140/RG.2.1.3810.7369

Qi F, Shi X-T, Liu F-F (2015b) An exponential representation for a function involving the gamma function and originating from the Catalan numbers. ResearchGate Res. doi:10.13140/RG.2.1.1086.4486

Qi F, Shi X-T, Liu F-F (2015c) An integral representation, complete monotonicity, and inequalities of the Catalan numbers, ResearchGate Technical report. doi:10.13140/RG.2.1.3754.4806

Qi F, Shi X-T, Liu F-F (2015d) Several formulas for special values of the Bell polynomials of the second kind and applications. ResearchGate Technical report. doi:10.13140/RG.2.1.3230.1927

Qi F, Shi X-T, Mahmoud M, Liu F-F (2015e) Schur-convexity of the Catalan-Qi function. ResearchGate Technical report. doi:10.13140/RG.2.1.2434.4802

Schilling RL, Song R, Vondraček Z (2012) Bernstein functions-theory and applications, 2nd ed. de Gruyter Studies in Mathematics, vol 37. Walter de Gruyter, Berlin. doi:10.1515/9783110269338

Shi X-T, Liu F-F, Qi F (2015) An integral representation of the Catalan numbers. Glob J Math Anal 3(3):130-133. doi:10.14419/gjma.v3i3.5055

Stanley R, Weisstein EW (2015) Catalan number, from MathWorld_a Wolfram web resource. http://mathworld.wolfram. com/CatalanNumber.html

Temme NM (1996) Special functions: an introduction to classical functions of mathematical physics. A Wiley-Interscience Publication, Wiley, New York. doi:10.1002/9781118032572

Vardi I (1991) Computational recreations in mathematica. Addison-Wesley, Redwood City

Wei C-F, Qi F (2015) Several closed expressions for the Euler numbers. J Inequal Appl 2015:219. doi:10.1186/ s13660-015-0738-9

Widder DV (1941) The laplace transform (Princeton mathematical series 6). Princeton University Press, Princeton

WikiPedia. Catalan number, from the free Encyclopedia. https://en.wikipedia.org/wiki/Catalan_number

\section{Submit your manuscript to a SpringerOpen ${ }^{\circ}$ journal and benefit from:}

- Convenient online submission

\section{- Rigorous peer review}

- Immediate publication on acceptance

- Open access: articles freely available online

High visibility within the field

- Retaining the copyright to your article

Submit your next manuscript at $\boldsymbol{\nabla}$ springeropen.com 